\title{
EMISSION-LINE ASYMMETRIES AND THE KINEMATICS OF THE NARROW-LINE REGION IN AGNS
}

\author{
Richard A. Shaw \\ Lick Observatory, University of California \\ Santa Cruz, CA 95064 \\ Michael M. De Robertis \\ Department of Physics, York University \\ 4700 Keele St., Downsview, Ont. M3J 1P3 CANADA
}

\begin{abstract}
We have obtained high signal-to-noise ratio CCD spectra at $\leq 150 \mathrm{~km} / \mathrm{s}$ resolution for 6 high-ionization Seyfert galaxies. We analyzed the profiles of the emission-lines over a wide range in both ionization potential (IP) and critical density $\left(\mathrm{N}_{c r}\right)$ in order to study the fundamental problem of cloud motion in the narrow-line region (NLR). Using the known correlations between FWHM and IP and/or $\mathrm{N}_{c r}$ for these galaxies, and assuming that the blueward profile asymmetries result from the combined effects of radially infalling or outflowing clouds and extinction within or between them, we deconvolve these effects by analyzing the correlation between emission-line asymmetries and both IP and $\mathrm{N}_{c r}$. We find fair to good correlations in the sense that lines with high IP and $\mathrm{N}_{c r}$ also tend to have high asymmetry, while lines with low IP and $\mathrm{N}_{c r}$ have low but usually nonzero asymmetry. Simulated emission-line profiles generated with a spherically-symmetric model of a NLR suggest that the extinction arises primarily within radially infalling clouds.
\end{abstract}

\section{Introduction}

A number of spectroscopic observations contribute to our knowedge of gas flow in AGNs. For example, the perponderance of blueward-asymmetric profiles of narrow emission-lines in AGNs has generally been interpreted as evidence for radial cloud motion coupled with differential extinction within the NLR. But the blueward asymmetry can result either from reddening between radially outflowing clouds, or from reddening within infalling clouds. By itself, then, narrow-line asymmetry provides only limited information on the kinematics of the NLR.

Recently, however, observations of LINERs and Seyfert $1 \mathrm{~s}$ and $2 \mathrm{~s}$ have revealed that line width often correlates with $N_{c r}$ and/or IP in AGNs. These findings strongly suggest that the NLR is stratified-that is, to first order, emission for a given ion arises in a shell-like volume surrounding the active nucleus. Further, the dispersion velocity and number density of the clouds must be inversely proportional to the distance from the continuum source. Combining these two observations, it is possible to determine fundamental NLR properties like the direction of cloud motion and the distribution of dust by comparing asymmetries among emission lines with different $I P$ and $N_{c r}$ in individual AGNs. The resolution of this question will lead to a more complete physical picture of the NLR, as well as indictate how 
reddening corrections should be applied to the broad-line and continuum radiation.

We observed only high-ionization AGNs-i.e., AGNs with emission lines that span a broad range in $I P$ and $N_{c r}$-and included only those galaxies where FWHM is known to correlate well with $I P$ and/or $N_{c r}$. We excluded galaxies with noticeable [Fe II] emission, or with very broad permitted lines, which would contaminate the narrow-line profiles. We obtained high signal-to-noise CCD spectra of 6 galaxies at high resolution $(2.5 \AA \mathrm{FWHM})$, as well as spectra of template galaxies in order to remove the effects of stellar absorption-line features.

\section{Results of This Study}

Most of the galaxies in our sample show good positive correlations between asymmetry and both $I P$ and $N_{c r}$-i.e., as $I P$ or $N_{c r}$ increases, so does asymmetry. We also note that the low $I P$ (or $N_{c r}$ ) lines have small, but usually non-zero asymmetries. Furthermore, the high IP lines generally have higher asymmetries at all levels than the low IP lines. Finally, we find some indication that lines with the highest $I P$ and/or $N_{c r}$ also show the lowest radial velocities, and vice versa, but the correlations are fairly weak.

The above results show that the highest $I P$ and $N_{c r}$ lines, which are formed near the center of activity, suffer the greatest extinction, and vice versa. The most straightforward interpretation is that the dust is largely confined to the NLR clouds themselves (which suggests a nearly constant gas-to-dust ratio in the NLR). If that is the case, then the clouds must be radially infalling in order to produce the observed bluewardly asymmetric profiles. However, it may be possible for the dust to be distributed between the clouds in a more complicated way, such that the observational conditions described above can still be met.

In order to investigate the effect of more complicated dust distributions, we calculated line profiles using spherically symmetric, numerical models of the NLR. We considered two limiting cases: one in which the extinction is confined completely to the inter-cloud medium, and the other in which the extinction arises entirely within infalling emission-line clouds. We modeled the NLR by uniformly filling a shell with many radially moving emission-line clouds and, for the case of outflow, with attenuating material according to either a constant, $1 / r$, or $1 / r^{2}$ density law. Here, the effective optical depth of the material depends upon the density distribution and path length through the NLR. We found that generating a profile with a sizeable asymmetry for the outer NLR required a dust distribution that is not centrally condensed. However, in order to produce large observed asymmetries in the inner NLR, the dust distribution must be centrally condensed. Thus, it is not possible to satisfy the observations for both the low and high critical density profiles simultaneously with this simple geometry.

In the case of inflow where the dust is confined to the clouds, the amount of attenuation is a function of the optical depth through the emitting cloud along the line of sight. Here we found no inconsistency, in that the low IP lines are the least asymmetric (but not necessarily zero), and vice versa. Evidently, extinction arising primarily within radially infalling emission-line clouds most easily explains the observed run of asymmetry with critical density. 